\title{
Dynamic extraction coupled on-line to liquid chromatography with a parallel sampling interface-a proof of concept for monitoring extraction kinetics
}

\author{
Mingzhe Sun $^{1} \cdot$ Said Al-hamimi $^{1} \cdot$ Margareta Sandahl $^{1} \cdot$ Charlotta Turner $^{1}$ \\ Received: 14 February 2019 / Revised: 28 March 2019 / Accepted: 12 April 2019 / Published online: 6 May 2019 \\ (C) The Author(s) 2019
}

\begin{abstract}
On-line hyphenation of extraction with chromatography has been explored in several different types of combinations. However, monitoring the complete process of a dynamic, continuous-flow extraction is not possible with any hyphenated system reported so far. The current work demonstrates that this challenging task can be effectively fulfilled by using a parallel sampling interface, which mimics the concept of comprehensive two-dimensional chromatography. In this study, pressurised hot water extraction was coupled on-line with ultra-high-performance liquid chromatography. The set-up was utilised in a kinetic study of dynamic pressurised hot water extraction of curcuminoids from turmeric powder. Compound-specific extraction curves were obtained, which clearly indicated the rate-limiting factors of the extraction processes under different conditions. Additionally, thermal degradation of curcumin during the extraction could also be demonstrated in some of the extractions.
\end{abstract}

Keywords Curcuminoids · Dynamic extraction · Liquid chromatography · On-line hyphenation · Parallel sampling · Turmeric powder

\section{Introduction}

As an important tool for both sample preparation and product purification, extraction techniques have been widely studied and utilised for many decades. Extraction can be performed in two different modes: static (batch) mode and dynamic (continuous flow) mode. Whilst the static mode generally requires simpler instrumentation and lower consumption of solvent, the dynamic process holds the advantage of enhanced mass transfer, faster kinetics and minimised compound degradation when the extraction is carried out under relatively harsh conditions [1]. In dynamic extraction processes of samples containing one or many major components, the compounds can be extracted at different times and different speeds, and a

Electronic supplementary material The online version of this article (https://doi.org/10.1007/s00216-019-01849-4) contains supplementary material, which is available to authorized users.

Charlotta Turner

charlotta.turner@chem.lu.se

1 Department of Chemistry, Centre for Analysis and Synthesis, Lund University, P.O. Box 124, 22100 Lund, Sweden different final yield is achieved depending on the specific extraction technique and conditions. In order to visualise the extraction profile including all compounds extracted, an on-line detector is usually added at the end of the extraction flow path $[2,3]$. Chemometric deconvolution of chromatographic data has been a useful tool in handling signals from co-eluting compounds [4]. This concept has also been applied to resolving extraction curves obtained from on-line detectors into compound or compound class-specific profiles [5]. One main limitation of the curve resolution approach is that analytes cannot have very similar spectra [5]. Hence, to further understand the kinetics of a dynamic extraction process or to obtain qualitative and quantitative information about the extract, chromatographic analysis is still an indispensable step of a complete work flow in most cases [6].

In most cases, chromatographic analysis in an extraction study is performed in two main modes: on-line or off-line. The main advantage of off-line analysis is the simple extract collection, as no special interface is required other than a container for extract collection. Off-line analysis, however, can be time-consuming and suffer from compound loss or degradation [7]. With a well-designed interface, on-line analysis can, to a large extent, eliminate the random errors in off-line 
collection and subsequent sample preparation procedures, as well as shorten the total run time. There have been several studies in the literature describing the hyphenation of different extraction techniques with on-line chromatographic analysis [8-12]. In most of these applications, the interface is a switching valve consisting of two positions. A loading module is installed in one of the positions for extract collection, and in the other position, a short capillary connection allows the mobile phase of the chromatography to flow through whilst no analysis is taking place. When a selected fraction of the extract is collected, the valve is switched to allow the collected extract to be flushed by the chromatography mobile phase into the analytical column for analysis. This type of set-up can be very useful for the analysis of one or multiple fractions during the extraction. However, since all systems reported so far only utilise one loading module on the interface, continuous monitoring of a real dynamic extraction process is not possible [8, 13]. This is because the extraction process must be temporarily put in static mode to avoid losing fractions of unanalysed extract when the loading module is switched off the extraction path to transfer the collected extract to the chromatographic column.

Our hypothesis is that this drawback can be resolved by online coupling of an extraction process with a chromatography system with an interface consisting of two parallel loading modules. The operation of this type of system resembles that of an on-line two-dimensional liquid chromatography (2D-LC) system. When 2D-LC is run in comprehensive mode, the firstdimension eluent is divided into a number of continual segments of the same time length that are called modulations. All modulations are sequentially transferred to the second dimension for fast separations. With a similar working principle, the proposed extraction-chromatography system can potentially allow the dynamic extraction flow to be collected by one sampling module whilst the other parallel sampling module is switched to the analytical flow for analysis. This would enable an on-line monitoring of specific extraction profiles of certain compounds or compound classes during a dynamic extraction process, without the need for sophisticated mathematical deconvolutions. So far, the usefulness of coupling a dynamic process with chromatography using the parallel sampling interface has only been touched upon in the specific case of monitoring drug release of a single compound [14].

To the best of our knowledge, parallel sampling has never been utilised in the on-line hyphenation of extraction with chromatography. In the present study, continuous-flow pressurised hot water extraction (PHWE) was hyphenated with ultra-high-performance liquid chromatography (UHPLC) with an interface consisting of two parallel sampling loops. As a proof of concept, this hyphenated PHWEUHPLC system was applied for the extraction kinetic study of three major curcuminoids from turmeric powder, where the advantages and limitations of the system were also discussed.

\section{Materials and methods}

\section{Chemicals}

Three curcuminoids, curcumin, demethoxycurcumin and bisdemethoxycurcumin, were purchased from Sigma Chemical Co. (St. Louis, MO, USA). Turmeric powder was purchased from a local supermarket. Ethanol (99.7\%) was obtained from Solveco (Rosersberg, Sweden). Acetonitrile was obtained from Scharlau (Barcelona, Spain). All organic solvents were of HPLC grade or above. All water used was from a Milli-Q water purification system with a UV unit.

\section{Apparatus}

The experiments were performed using a home-built PHWEUHPLC system. The PHWE system used is similar to one previously described with slight modifications [15]. Water was pre-heated to $70^{\circ} \mathrm{C}$ before being pumped into a $\mathrm{GC}$ oven held at different temperatures under which the extraction took place. The GC oven contained the extraction cell and a long pre-heating coil installed before the inlet of the extraction cell. The volume of the extraction vessel used was $3 \mathrm{~mL}$. An additional post-extraction make-up ethanol flow was mixed with the $\mathrm{H}_{2} \mathrm{O}$ extract before cooling in order to eliminate any precipitation occurring during the cooling process. The UHPLC part consisted of a degasser (G4225A, Agilent Technologies), a binary pump (G4220A, Agilent Technologies), a thermostated column compartment (G1316C, Agilent Technologies) and a diode array detector (G4212B, Agilent Technologies). As for the interface coupling PHWE with UHPLC, a flexible cube module was used (G4227, Agilent Technologies), in which an 8-port-2position switching valve (5067-4214, Agilent Technologies) was installed with two identical $60-\mu \mathrm{L}$ collection loops. A flow splitter was installed between the outlet of the PHWE and the interface (600-PO10-06, Supelco Analytical). For each specific PHWE flow rate after the cooling compartment, a specific split ratio (set by the adjustable flow splitter) was used to allow the collection loop to be filled in one analysis time. The splitting ratios provided by the splitter were verified by collecting the two splitting flows and weighing. The scheme of the PHWE-UHPLC system is shown in Fig. 1. When the interface was at position A, collection loop a is responsible for collecting the fraction of the PHWE extract, whilst the collected extract in loop $\mathrm{b}$ is being flushed by the UHPLC flow into the column for analysis. After one analysis time, the interface switches to position $\mathrm{B}$, where the roles of loops a and $\mathrm{b}$ also switch. An Agilent Openlab CDS Chemstation C.01.07 software was used for system control. Agilent Chemstation software was used for data processing. 


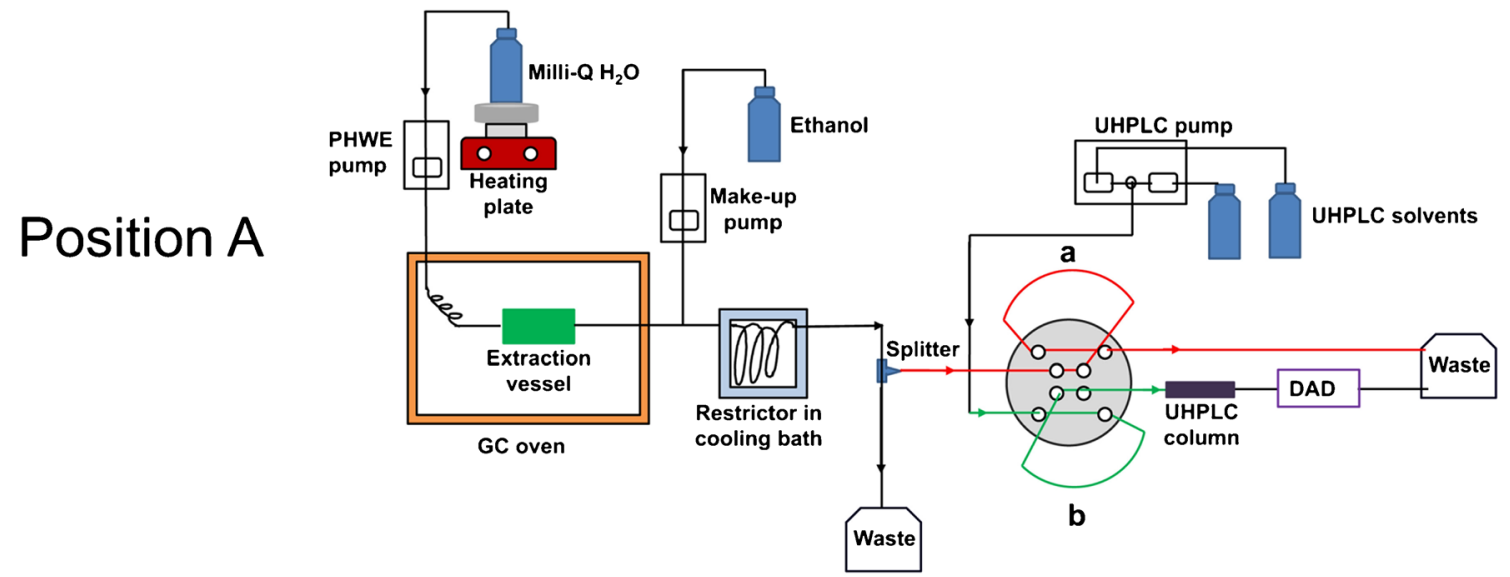

Position B

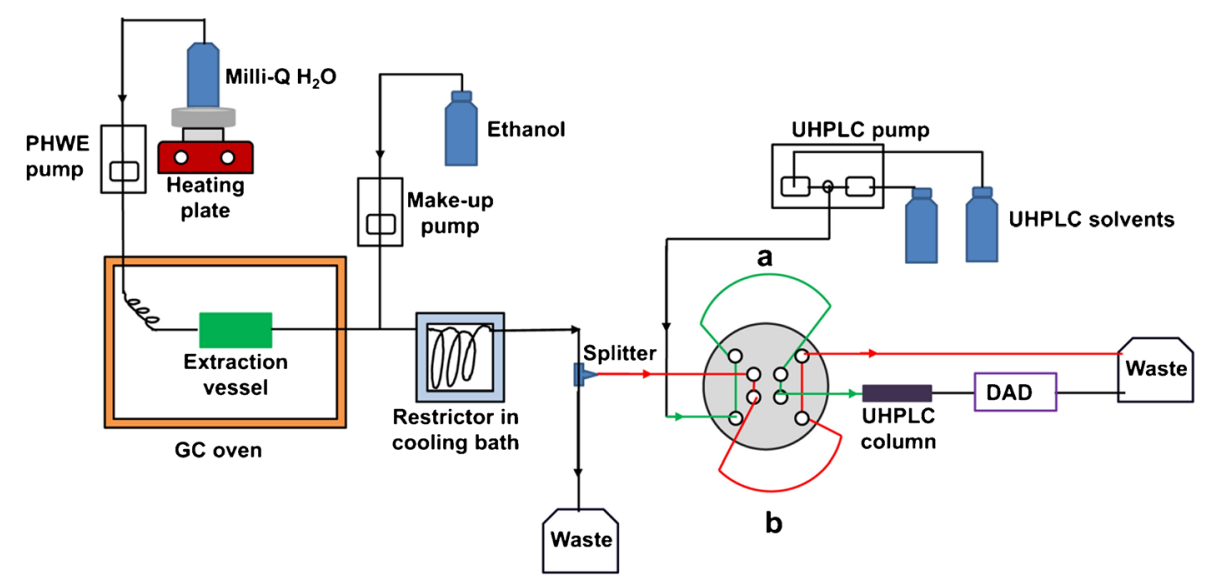

Fig. 1 Scheme of the PHWE-UHPLC system

\section{Column screening and method tuning of the liquid chromatography part}

The UHPLC part was connected to the auto-sampler and used as stand-alone UHPLC for column screening and method tuning. Four different columns of the same dimensions but different selectivities available in the lab were screened for the separation of major compounds in turmeric PHWE extract: Agilent Poroshell 120 Eclipse-Cyano (EC-CN, 4.6× $50 \mathrm{~mm}, 2.7 \mu \mathrm{m}$ ); Agilent Poroshell 120 Eclipse-C18 (ECC18, $4.6 \times 50 \mathrm{~mm}, 2.7 \mu \mathrm{m}) ;$ Agilent Poroshell 120 Pentafluorophenyl (PFP, $4.6 \times 50 \mathrm{~mm}, 2.7 \mu \mathrm{m})$ and Agilent Poroshell 120 Phenyl-hexyl (P-H, $4.6 \times 50 \mathrm{~mm}, 2.7 \mu \mathrm{m})$. An extraction of turmeric powder was performed with the same PHWE part in the on-line system. Milli-Q water was preheated to $70{ }^{\circ} \mathrm{C}$ before being pumped as an extraction solvent. The extraction vessel was heated inside the GC oven to the set temperature and maintained at the temperature for $1 \mathrm{~min}$ after the sample was loaded. The dynamic extraction was then started at $90{ }^{\circ} \mathrm{C}$ with a water flow rate of $0.5 \mathrm{~mL} / \mathrm{min}$ and $0.1 \mathrm{~mL} / \mathrm{min}$ ethanol make-up flow for $1 \mathrm{~h}$. The extract was collected in a 50-mL tube and placed under gentle nitrogen flow in dark for solvent evaporation overnight. The extract after solvent removal was then re-dissolved in ethanol and was further used for screening and optimization experiments. The injection volume was $5 \mu \mathrm{L}$. The separation was monitored with DAD at $280 \mathrm{~nm}$ and $430 \mathrm{~nm}$ with a $20-\mathrm{Hz}$ sampling frequency. The column temperature was set at $30^{\circ} \mathrm{C}$. The flow rate was set at $2.5 \mathrm{~mL} / \mathrm{min}$. The mobile phase consisted of $\mathrm{A}$, water $(0.3 \%$ acetic acid) and $\mathrm{B}$, acetonitrile ( $0.3 \%$ acetic acid). The gradient started with $20 \% \mathrm{~B}$ and then ramp up to $90 \%$ in $1.5 \mathrm{~min}$. Then, the gradient was maintained at $90 \%$ for $0.5 \mathrm{~min}$ before decreasing to the starting composition. The identification of the three curcuminoids was performed by injecting single-standard solutions of each curcuminoid $(50 \mu \mathrm{g} / \mathrm{mL})$ in a mix of water and ethanol (50:50, vol\%) prepared and stored in dark.

\section{UHPLC-QTOF/MS analysis}

A fast method was used to confirm the identification of major compounds in the turmeric extract. Briefly, $2 \mu \mathrm{L}$ of the extract was injected onto a Waters Acquity UPLC BEH-C18 column $(100 \mathrm{~mm} \times 2.1 \mathrm{~mm}, 1.7 \mu \mathrm{m}$; Waters Corporation, Milford, MA) using an ACQUITY UPLC® system (Waters Corporation, Milford, MA). The mobile phase consisted of (A) $\mathrm{H}_{2} \mathrm{O}$ and (B) acetonitrile, both containing $0.1 \%(\mathrm{v} / \mathrm{v})$ formic acid. The column temperature was $40^{\circ} \mathrm{C}$ and the flow 
rate $400 \mu \mathrm{L} / \mathrm{min}$. Gradient elution was used starting at $40 \% \mathrm{~B}$, then increasing from 40 to $70 \% \mathrm{~B}$ over 0 to $3 \mathrm{~min}, 70$ to $95 \%$ $\mathrm{B}$ over 3 to $3.5 \mathrm{~min}$, and kept at $95 \% \mathrm{~B}$ for $1 \mathrm{~min}$, and finally returned to initial conditions, for $2 \mathrm{~min}$. MS was performed on a Xevo ${ }^{\text {TM }} \mathrm{G} 2$ quadrupole time of flight (QTOF) (Waters MS Technologies, Manchester, UK). A mass spectrometer was scanning from 100 to $1000 \mathrm{~m} / \mathrm{z}$, the cone voltage was set to $35 \mathrm{~V}$ and the capillary voltage was set to $2.5 \mathrm{~V}$ in negative ESI mode. The desolvation gas flow rate was $600 \mathrm{~L} / \mathrm{h}$ at a temperature of $500{ }^{\circ} \mathrm{C}$ and the cone gas flow rate was $20 \mathrm{~L} / \mathrm{h}$. The source temperature was $120^{\circ} \mathrm{C}$.

\section{Final hyphenated system operating parameters}

Milli-Q water was pre-heated to $70^{\circ} \mathrm{C}$ before being pumped as the extraction solvent. The extraction vessel was heated inside the GC oven to the set temperature and maintained at the temperature for $1 \mathrm{~min}$ after the sample was loaded. The dynamic extraction was started right afterwards with water pumped at a specific flow rate for the whole experiment. The make-up ethanol flow was maintained at $0.1 \mathrm{~mL} / \mathrm{min}$ for all experiments. Two temperatures, 90 and $140{ }^{\circ} \mathrm{C}$, and two flow rates, 0.5 and $0.8 \mathrm{~mL} / \mathrm{min}$, were used in the kinetic study. Each experiment was repeated three times. The column temperature was set at $33{ }^{\circ} \mathrm{C}$. The flow rate was set at $3.2 \mathrm{~mL} /$ min. The mobile phase consisted of $\mathrm{A}$, water $(0.3 \%$ acetic acid) and $\mathrm{B}$, acetonitrile $(0.3 \%$ acetic acid). The gradient started with $50 \% \mathrm{~B}$ and then ramp up to $56 \%$ in $0.5 \mathrm{~min}$. Then, acetonitrile increased to $95 \%$ in $0.3 \mathrm{~min}$. The gradient was maintained at $95 \%$ for $0.15 \mathrm{~min}$ before rapidly decreasing to the starting composition for re-equilibration for $0.25 \mathrm{~min}$. Each analysis lasted $1.2 \mathrm{~min}$ in total. Calibration curves of the three curcuminoids were obtained by injecting standard solutions in a mix of water and ethanol (50:50, vol\%) of a series of concentrations $(1 \mu \mathrm{g} / \mathrm{mL}$ to $333 \mu \mathrm{g} / \mathrm{mL}$ ) using the same UHPLC in the hyphenated system under the same optimised chromatographic conditions listed above.

\section{Results and discussion}

The root of the turmeric (Curcuma longa L.) plant is widely used in food, traditional medicine and textile colourants in south Asia [16]. The principal components of turmeric are curcumin and its demethoxy and bisdemethoxy derivatives (see Electronic Supplementary Material (ESM) Fig. S1), which have been proven to exhibit various beneficial health and medicinal effects [17, 18]. The use of hot water or pressurised hot water as extraction solvent has been widely applied in many extraction applications including the extraction of curcuminoids from turmeric [16, 19-21]. In the current study, PHWE of three major curcuminoids from a commercial turmeric power under different extraction conditions was performed as a proof of concept of the new extractionchromatography system coupled using parallel sampling.

\section{Tuning of the liquid chromatography separation}

Figure 2 (left side) shows column screening chromatograms of a turmeric PHWE extract detected at $430 \mathrm{~nm}$. The $\mathrm{CN}$ column gave apparently the worst performance with a wide peak and hardly any separation of the three curcuminoids. Among the other three screened columns, the P-H and C18 columns exhibited similar resolution of the targeted compounds ( $\mathrm{Rs}=0.95,0.90$ and $0.97,0.93$ for the two pairs of peaks separated on $\mathrm{P}-\mathrm{H}$ and $\mathrm{C} 18$ columns, respectively). However, the P-H column provided narrower peak width and better peak shape. Another aspect that should be taken into consideration in the selection of the UHPLC column is the analysis time. Chromatograms at $280 \mathrm{~nm}$ revealed that a wider profile of compounds was extracted (Fig. 2, right side). Peaks eluting before the curcuminoids were likely composed of a variety of phenolic compounds, flavonoids and their glycosides [22]. Two major peaks that eluted late in the chromatograms (peaks 4 and 5) consisted of mainly two phospholipids, as confirmed by performing UHPLC-QTOF/MS analysis. It is obvious that for these relatively less polar compounds, the retention was stronger on the C18 column than on the P-H column. Thus, the usage of the $\mathrm{C} 18$ column would require longer analysis time in order to avoid un-eluted compounds interfere with the analysis of the next collected extract fraction, which is similar to the wrap-around issues in twodimensional liquid chromatography [23]. The P-H column was therefore chosen for optimization as shorter analysis time allows higher sampling frequency, which adds more details into the final extraction profiles generated. With the final optimised UHPLC method, the analysis time was shortened to $1.2 \mathrm{~min}$. The limits of detection and quantification ( 3 and 10 times signal-to-noise ratio) were $25 \mathrm{ng} / \mathrm{mL}$ and $100 \mathrm{ng} / \mathrm{mL}$ respectively for the three curcuminoids. The absorption behaviour of the curcuminoids was linear from approximately $100 \mathrm{ng} / \mathrm{mL}$ to $400 \mu \mathrm{g} / \mathrm{mL}$.

\section{Dynamic extraction and analysis of curcuminoids from turmeric}

If an extraction process is coupled with an on-line UV detector, a general extraction curve can usually be obtained showing the UV absorption of the eluting extract at different time points (Fig. 3a). However, information regarding specific compounds can be very difficult to acquire from the general extraction curve. With the system reported in this work, the PHWE extract was divided into a series of short fractions and each fraction was sequentially collected and analysed by UHPLC. The hyphenated system can thus produce a series of continual short LC chromatograms monitoring the whole 
Fig. 2 LC column screening results on four columns and two wavelengths. a-d Screening chromatograms for different columns monitored at $430 \mathrm{~nm}$. e, f Screening chromatograms for $\mathrm{C} 18$ and $\mathrm{P}-\mathrm{H}$ columns monitored at $280 \mathrm{~nm}$ (for chromatographic conditions, see the "Materials and methods" section). Peaks 1, 2 and 3 are bisdemethoxycurcumin, demethoxycurcumin and curcumin respectively. Peaks 4 and 5 are two phospholipids
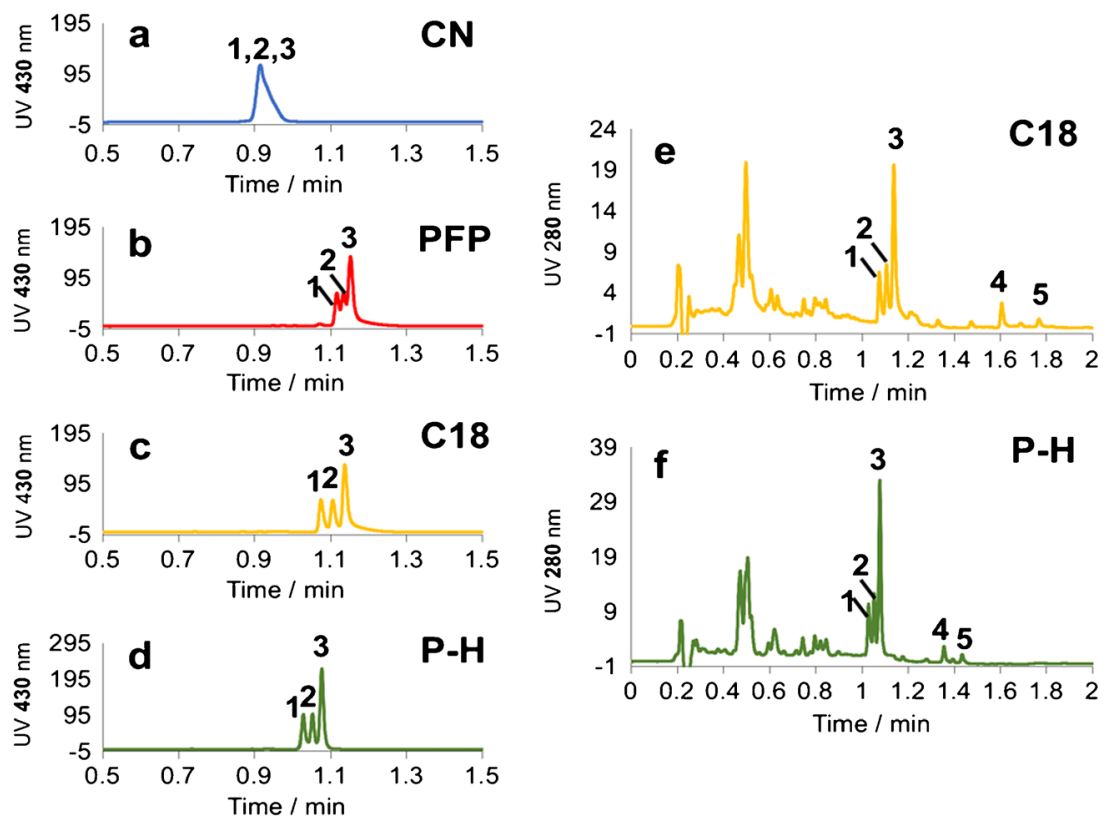

process (Fig. 3b). These chromatograms can be used for three main purposes: (1) to determine the content of compounds in the extract at a certain time; (2) to generate a compoundspecific extraction yield vs. extraction time curve in order to understand the kinetics of the extraction (Fig. 3c); and (3) to monitor the occurrence of possible degradation or other reactions taking place during the extraction, i.e. extraction bias.

Figure 4 shows the specific profiles of major compounds in the extract during different stages of the extraction processes under different temperatures. This type of data can be used for several purposes, for instance to quantify specific molecular species in complex sample mixtures; to obtain extraction kinetic data for selected compounds to explore selectivity evolvement during the extraction; and to assess extraction bias in terms of molecular degradation/reaction. For example, it can be noted from Fig. 4 that when the extraction was carried out at $90{ }^{\circ} \mathrm{C}$, the relative amount of extracted curcumin (peak area) in comparison with the other two curcumin derivatives kept increasing. Interestingly, a completely opposite tendency was observed when the extraction temperature was set at $140{ }^{\circ} \mathrm{C}$; the ratio between extracted curcumin and the other two derivatives kept decreasing rapidly during the whole extraction process. This could be the results of compound degradation as it has long been known that curcuminoids, especially curcumin, have poor thermal stability in an aqueous environment [24, 25].

As illustrated in Fig. 3, extraction curves can be constructed for specific compounds. Figure 5 shows extraction curves for the three investigated curcuminoids, obtained at different extraction temperatures $\left(90\right.$ and $\left.140{ }^{\circ} \mathrm{C}\right)$ and flow rates $(0.5$ and $0.8 \mathrm{~mL} / \mathrm{min}$ ). These curves can be used to achieve information about the initial extraction rate, the overall progress of the extraction, as well as the final extractable amount given a
Fig. 3 a-c A general scheme demonstrating how UHPLC chromatograms of all fractions in a PHWE-UHPLC run are used to generate a compound-specific extraction curve

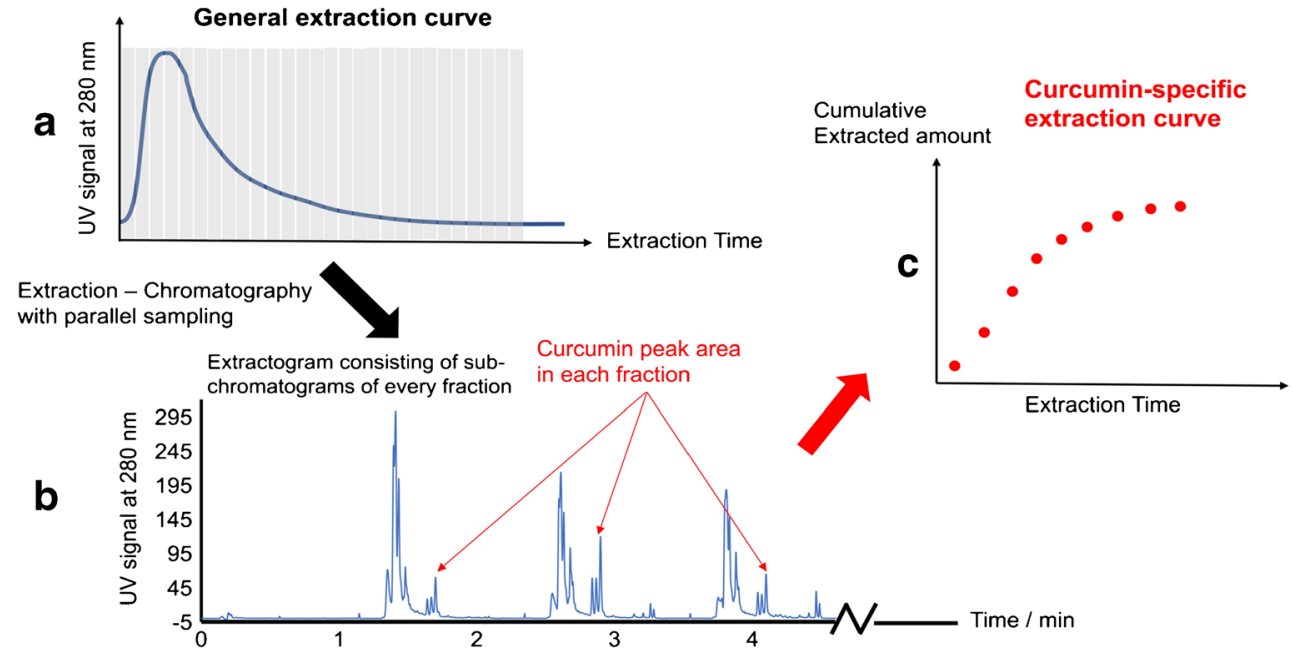




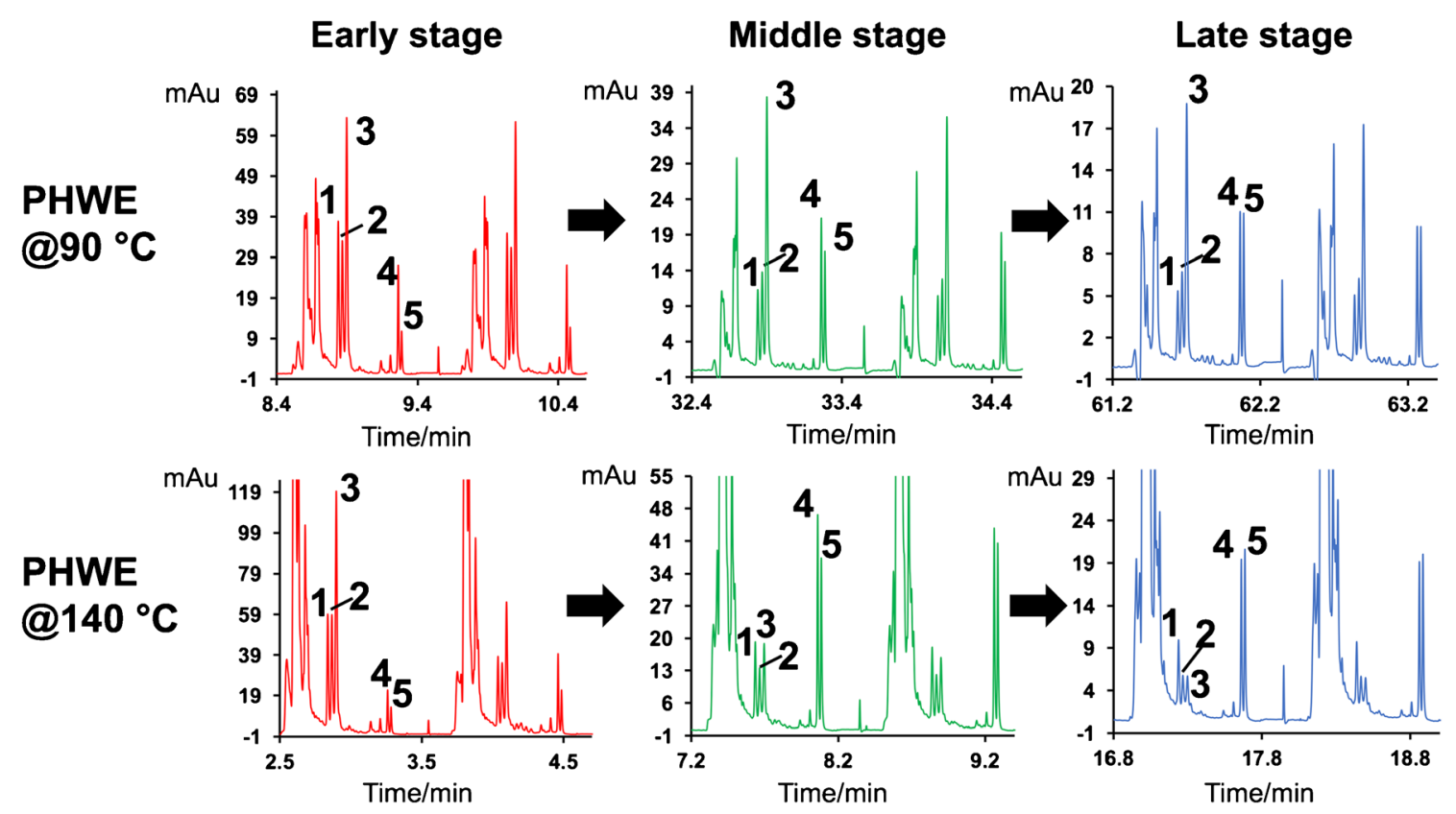

Fig. 4 Compound profiles in extracts at different extraction times for two different extraction temperatures, showing two consecutive fractions per chromatogram. Peaks 1, 2 and 3 are bisdemethoxycurcumin, demethoxycurcumin and curcumin respectively. Peaks 4 and 5 are two phospholipids

certain extraction condition. The progression of the extraction can be described as a function of extraction time or solvent volume. For instance, as shown in Fig. 5 (lower part) where the extracted amount is expressed as a function of solvent volume, it is clear that the initial stage of the extractions conducted at $90{ }^{\circ} \mathrm{C}$ gives overlapping extraction curves, i.e. the extraction rate is mainly solubility-controlled under this condition. After ca. 9-14 $\mathrm{mL}$ of extraction solvent used, the extraction curves deviate, and the extraction rate is more controlled by mass transfer.
For the higher extraction temperature $\left(140{ }^{\circ} \mathrm{C}\right)$, the initial extraction rate is significantly faster for all three compounds compared with extractions conducted at $90^{\circ} \mathrm{C}$. Also, the extraction curves at $140^{\circ} \mathrm{C}$ were close to levelling out at $80 \mathrm{~min}$. However, it can be observed from the trends of the extraction curves that the final extracted amount would be lower than that using the lower temperature. This is true even though the extraction at the lower temperature is far from being a complete extraction at $80 \mathrm{~min}$. A higher extraction temperature enables both higher solubility and faster mass transfer;
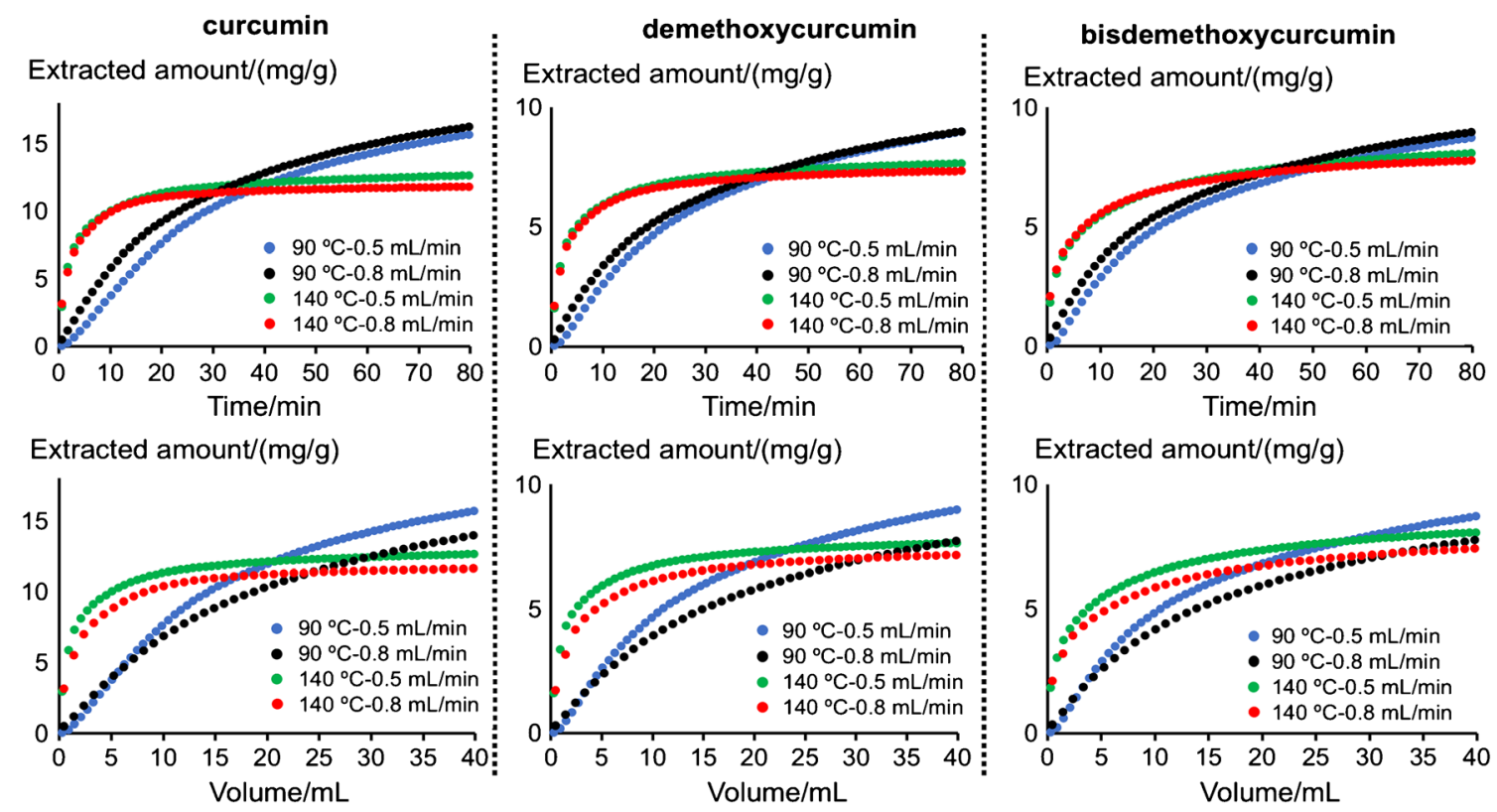

Fig. 5 Extraction curves for three curcuminoids under different conditions (see the "Materials and methods" section for UHPLC conditions) 
however, as shown by the overlapping curves when plotted vs. extraction time, the extraction is mainly mass transfer limited. Figure 5 also shows that the downside of a higher extraction temperature like $140^{\circ} \mathrm{C}$ is thermal degradation, as clearly seen for all three curcuminoids. This is in accordance with a previous study on static extraction [16]. Moreover, a comparison with the static extraction study also revealed that the consumption of solvent could be reduced and extraction speed accelerated if some extraction parameters are optimised, such as particle size of turmeric and pre-treatment of the powder.

Obtained results can also be plotted as extractograms (contour plots), exemplified with PHWE at $90^{\circ} \mathrm{C}$ and $140{ }^{\circ} \mathrm{C}$ with $0.5 \mathrm{~mL} / \mathrm{min}$ (see ESM, Figs. S2 to S4). Such figures can be useful to illustrate extractability of compounds under different extraction conditions.

We propose that the hyphenated dynamic extractionchromatography system using parallel sampling described in this study enables the quantification of important factors in extraction such as initial extraction rate, solubility vs. mass transfer-controlled rate-limiting stages of the extraction, as well as potential degradation of specific compounds during the extraction, in addition to quantification of total extracted amount (see Table 1 for calculated initial extraction rates of all three curcuminoids under all experimental conditions). Again, it is clear that for the initial extraction rate, constants at the lower temperature are very similar when calculated per solvent volume unit, demonstrating solubility-controlled extraction kinetics, whilst for the higher temperature, the extraction rate constants are similar when calculated per time unit, demonstrating an example of mass transfer-controlled extraction kinetics, in this case also combined with degradation. Here, when plotted per solvent volume unit, an obvious extract dilution takes place with the higher flow rate.

Compilation of the data (as shown in Fig. 5) requires the retention time of the analyte peaks to be highly repeatable. The repeatability and reproducibility of the system were accessed with curcumin peaks from 10 randomly selected
LC runs in 1 day and curcumin peaks from six randomly selected LC runs in six different days. The within-day relative standard deviation of retention time was determined to be $0.34 \%$ and the between-day relative standard deviation of retention time was determined to be $1.2 \%$.

\section{Comparison with traditional off-line analysis}

In order to verify the reliability of the extraction curves obtained with the developed on-line system, extractions were performed with the same PHWE system. The extract was collected periodically in fractions, which were followed by off-line analysis using the same UHPLC system included in the hyphenated set-up. As can be seen in Fig. 6, the traditional off-line analysis yielded similar extraction curves as the corresponding curves generated from the on-line system. Meanwhile, much more detailed information regarding the compound extracted during the extraction process can be acquired using the on-line system with all the chromatographic analysis completed along with the extraction. Even though data processing of the large number of short chromatograms obtained can be time-consuming, the on-line system eliminated the extra sample preparation and chromatographic analysis time in the off-line approach.

\section{Limitations with the system and future aspects}

In a traditional 2D-LC system, samples are commonly filtered before the injection and guard columns are usually installed before the first-dimension column. However, in a similar system coupling extraction and chromatography presented in this work, the extract enters the LC column directly. Under this circumstance, guard columns should instead be used before the column to filter out particles and contaminants, which in the meantime increases the gradient delay volume and elution time of strongly

Table 1 Initial extraction rate under all experimental conditions, calculated both as a function of time and as a function of solvent volume. Data obtained in the range where the extraction curves have a $R^{2}$ value higher than 0.99

\begin{tabular}{|c|c|c|c|c|}
\hline & \multicolumn{4}{|c|}{ Initial rate as a function of time $\left(\mathrm{mg} /\left(\mathrm{g}^{*} \mathrm{~min}\right)\right)$} \\
\hline & $90{ }^{\circ} \mathrm{C}$ & $90{ }^{\circ} \mathrm{C}$ & $140^{\circ} \mathrm{C}$ & $140^{\circ} \mathrm{C}$ \\
\hline & $0.5 \mathrm{~mL} / \mathrm{min}$ & $0.8 \mathrm{~mL} / \mathrm{min}$ & $0.5 \mathrm{~mL} / \mathrm{min}$ & $0.8 \mathrm{~mL} / \mathrm{min}$ \\
\hline Curcumin & 0.36 & 0.59 & 1.75 & 1.61 \\
\hline Demethoxycurcumin & 0.26 & 0.38 & 1.13 & 1.03 \\
\hline \multirow[t]{3}{*}{ Bisdemethoxycurcumin } & 0.29 & 0.41 & 0.81 & 0.76 \\
\hline & \multicolumn{4}{|c|}{ Initial rate as a function of solvent volume $(\mathrm{mg} /(\mathrm{g} * \mathrm{~mL}))$} \\
\hline & $\begin{array}{l}90{ }^{\circ} \mathrm{C} \\
0.5 \mathrm{~mL} / \mathrm{min}\end{array}$ & $\begin{array}{l}90{ }^{\circ} \mathrm{C} \\
0.8 \mathrm{~mL} / \mathrm{min}\end{array}$ & $\begin{array}{l}140^{\circ} \mathrm{C} \\
0.5 \mathrm{~mL} / \mathrm{min}\end{array}$ & $\begin{array}{l}140^{\circ} \mathrm{C} \\
0.8 \mathrm{~mL} / \mathrm{min}\end{array}$ \\
\hline Curcumin & 0.72 & 0.73 & 3.50 & 2.01 \\
\hline Demethoxycurcumin & 0.52 & 0.48 & 2.26 & 1.29 \\
\hline Bisdemethoxycurcumin & 0.58 & 0.52 & 1.62 & 0.95 \\
\hline
\end{tabular}




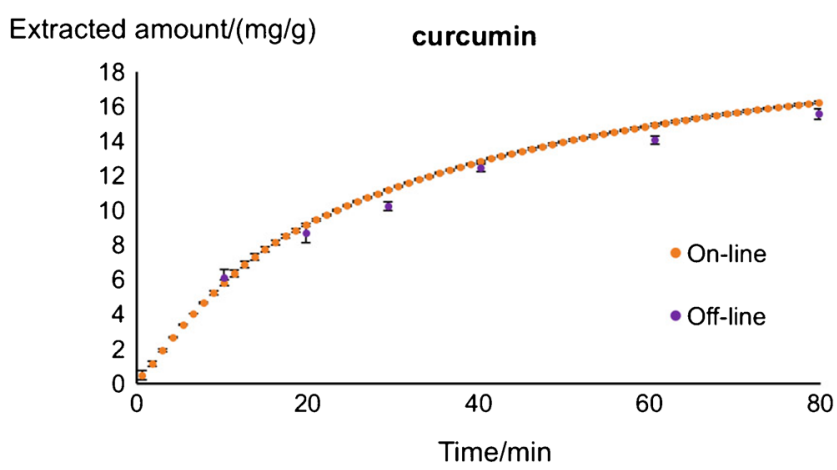

Extracted amount $/(\mathrm{mg} / \mathrm{g}) \quad$ demethoxycurcumin

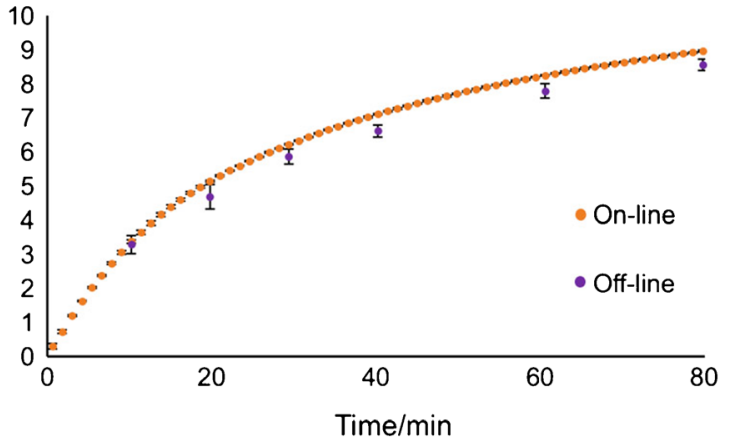

Extracted amount/(mg/g) bisdemethoxycurcumin

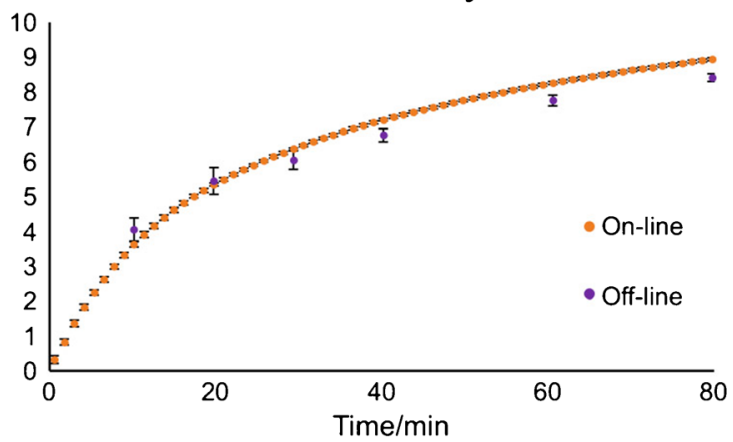

Fig. 6 Comparison between the proposed on-line approach and the offline approach. Extraction was performed under $90^{\circ} \mathrm{C}$ with $0.8 \mathrm{~mL} / \mathrm{min}$ as extraction flow rate (see the "Materials and methods" section for UHPLC conditions). Error bar represents standard deviation of a specific point obtained with 3 repeated experiments

retained compounds. For samples containing relatively high numbers of targeted compounds to be extracted, the analysis time should be inevitably prolonged to achieve a decent separation. This leads to lower sampling frequency of the interface and possible loss of information.

In order to improve and apply the parallel sampling interfacing concept to other types of extractions, future work should be focused on two aspects: (1) The interface design has to be modified according to the specific extraction technique. For example, sampling loops could be replaced by solid phase traps to effectively capture and concentrate the compounds from the extraction process. (2) Apply mass spectrometry detection after chromatography to further increase the resolving power and enable compound identification.

\section{Conclusions}

Dynamic pressurised hot water extraction was coupled on-line with ultra-high-performance liquid chromatography using a parallel sampling interface and applied as a proof of concept to a compound-specific kinetic study. The profiles of compounds extracted at different times of the extraction were monitored. Extraction curves for the curcuminoids demonstrated that solubility was the main initial controlling factor for extraction at $90^{\circ} \mathrm{C}$, whilst extractions conducted at $140^{\circ} \mathrm{C}$ enabled faster extraction rates although the extracted amount was hampered by severe thermal degradation. This study showed for the first time that coupling extraction with chromatography with parallel sampling can be very useful for the study of the extraction behaviours of compounds or compound classes in a dynamic extraction process. The obtained results were also confirmed by an off-line method.

Funding information This work is financially supported by the Swedish Research Council Formas (2016-00604) and the Swedish Foundation for Strategic Research (SSF, RBP 14-0052).

\section{Compliance with ethical standards}

Conflict of interest The authors declare that they have no conflict of interest.

Open Access This article is distributed under the terms of the Creative Commons Attribution 4.0 International License (http:// creativecommons.org/licenses/by/4.0/), which permits unrestricted use, distribution, and reproduction in any medium, provided you give appropriate credit to the original author(s) and the source, provide a link to the Creative Commons license, and indicate if changes were made.

\section{References}

1. Raynie DE. Modern extraction techniques. Anal Chem. 2010;82(12):4911-6. https://doi.org/10.1021/ac101223c.

2. Morales-Munoz S, Luque-Garcia JL, de Castro MDL. Pressurized hot water extraction with on-line fluorescence monitoring: a comparison of the static, dynamic, and static-dynamic modes for the removal of polycyclic aromatic hydrocarbons from environmental solid samples. Anal Chem. 2002;74(16):4213-9. https://doi.org/10. $1021 / \mathrm{ac} 0257288$

3. Amador-Hernandez J, De Castro MDL. On-line detection for supercritical-fluid extraction. J Biochem Biophys Methods. 2000;43(13):329-43. https://doi.org/10.1016/S0165-022x(00)00097-X.

4. Sinanian MM, Cook DW, Rutan SC, Wijesinghe DS. Multivariate curve resolution-alternating least squares analysis of highresolution liquid chromatography-mass spectrometry data. Anal Chem. 2016;88(22):11092-9. https://doi.org/10.1021/acs. analchem.6b03116.

5. Abrahamsson V, Jumaah F, Turner C. Continuous multicomponent quantification during supercritical fluid extraction applied to microalgae using in-line UV/Vis absorption spectroscopy and online evaporative light scattering detection. J Supercrit Fluids. 2018;131:157-65. https://doi.org/10.1016/j.supflu.2017.09.014. 
6. Ferreira VG, Leme GM, Cavalheiro AJ, Funari CS. Online extraction coupled to liquid chromatography analysis (OLELC): eliminating traditional sample preparation steps in the investigation of solid complex matrices. Anal Chem. 2016;88(17):8421-7. https://doi. org/10.1021/acs.analchem.6b02388.

7. Hyotylainen T, Riekkola ML. Approaches for on-line coupling of extraction and chromatography. Anal Bioanal Chem. 2004;378(8): 1962-81. https://doi.org/10.1007/s00216-004-2545-x.

8. Sanchez-Camargo AD, Parada-Alfonso F, Ibanez E, Cifuentes A. On-line coupling of supercritical fluid extraction and chromatographic techniques. J Sep Sci. 2017;40(1):213-27. https://doi.org/ 10.1002/jssc. 201601040.

9. Lucci P, Nunez O. On-line solid-phase extraction for liquid chromatography-mass spectrometry analysis of pesticides. J Sep Sci. 2014;37(20):2929-39. https://doi.org/10.1002/jssc. 201400531.

10. Zambonin CG. Coupling solid-phase microextraction to liquid chromatography. A review. Anal Bioanal Chem. 2003;375(1):7380. https://doi.org/10.1007/s00216-002-1623-1.

11. Hyotylainen T, Andersson T, Hartonen K, Kuosmanen K, Riekkola ML. Pressurized hot water extraction coupled on line with LC-GC: determination of polyaromatic hydrocarbons in sediment. Anal Chem. 2000;72(14):3070-6. https://doi.org/10.1021/ac0002821.

12. Chen SY, Urban PL. On-line monitoring of Soxhlet extraction by chromatography and mass spectrometry to reveal temporal extract profiles. Anal Chim Acta. 2015;881:74-81. https://doi.org/10. 1016/j.aca.2015.05.003.

13. Pan JL, Zhang CJ, Zhang ZM, Li GK. Review of online coupling of sample preparation techniques with liquid chromatography. Anal Chim Acta. 2014;815:1-15. https://doi.org/10.1016/j.aca.2014.01. 017.

14. Helle A, Hirsjarvi S, Peltonen L, Hirvonen J, Wiedmer SK, Hyotylainen T. Novel, dynamic on-line analytical separation system for dissolution of drugs from poly (lactic acid) nanoparticles. J Pharm Biomed Anal. 2010;51(1):125-30. https://doi.org/10.1016/ j.jpba.2009.08.021.

15. Liu JY, Sandahl M, Sjoberg PJR, Turner C. Pressurised hot water extraction in continuous flow mode for thermolabile compounds: extraction of polyphenols in red onions. Anal Bioanal Chem. 2014;406(2):441-5. https://doi.org/10.1007/s00216-013-7370-7.

16. Kiamahalleh MV, Najafpour-Darzi G, Rahimnejad M, Moghadamnia AA, Kiamahalleh MV. High performance curcumin subcritical water extraction from turmeric (Curcuma longa L.). J
Chromatogr B. 2016;1022:191-8. https://doi.org/10.1016/j. jchromb.2016.04.021.

17. Duvoix A, Blasius R, Delhalle S, Schnekenburger M, Morceau F, Henry E, et al. Chemopreventive and therapeutic effects of curcumin. Cancer Lett. 2005;223(2):181-90. https://doi.org/10. 1016/j.canlet.2004.09.041.

18. Surh YJ. Anti-tumor promoting potential of selected spice ingredients with antioxidative and anti-inflammatory activities: a short review. Food Chem Toxicol. 2002;40(8):1091-7. https://doi.org/ 10.1016/S0278-6915(02)00037-6.

19. Plaza M, Turner C. Pressurized hot water extraction of bioactives. TrAC Trends Anal Chem. 2015;71:39-54. https://doi.org/10.1016/ j.trac.2015.02.022.

20. Euterpio MA, Cavaliere C, Capriotti AL, Crescenzi C. Extending the applicability of pressurized hot water extraction to compounds exhibiting limited water solubility by $\mathrm{pH}$ control: curcumin from the turmeric rhizome. Anal Bioanal Chem. 2011;401(9):2977-85. https://doi.org/10.1007/s00216-011-5383-7.

21. Hawthorne SB, Yang Y, Miller DJ. Extraction of organic pollutants from environmental solids with subcritical and supercritical water. Anal Chem. 1994;66(18):2912-20. https://doi.org/10.1021/ ac00090a019.

22. Grumezescu AM, Holban AM. Natural and artificial flavoring agents and food dyes. Handbook of food bioengineering, vol 7. London: Academic Press, an imprint of Elsevier; 2018.

23. Pirok BWJ, Gargano AFG, Schoenmakers PJ. Optimizing separations in online comprehensive two-dimensional liquid chromatography. J Sep Sci. 2018;41(1):68-98. https://doi.org/10.1002/jssc. 201700863.

24. Heffernan C, Ukrainczyk M, Gamidi RK, Hodnett BK, Rasmuson AC. Extraction and purification of curcuminoids from crude curcumin by a combination of crystallization and chromatography. Org Process Res Dev. 2017;21(6):821-6. https://doi.org/10.1021/ acs.oprd.6b00347.

25. Esatbeyoglu T, Ulbrich K, Rehberg C, Rohn S, Rimbach G. Thermal stability, antioxidant, and anti-inflammatory activity of curcumin and its degradation product 4-vinyl guaiacol. Food Funct. 2015;6(3):887-93. https://doi.org/10.1039/c4fo00790e.

Publisher's note Springer Nature remains neutral with regard to jurisdictional claims in published maps and institutional affiliations. 